\title{
Robustness of Front-Wheel-Steered Vehicles with the Angle Controllers Connected to Actual Lane Command
}

\author{
Bing-Fei Wu, and Shih-Meng Chang, Members, IEEE
}

\begin{abstract}
This study analyzes front wheels steering (FWS) vehicles using results obtained from two unmanned cars: Unmanned Car I (UCI) and Unmanned Car II (UCII). The vehicle model integrates the lane angle derived from the translational system such that data on the lateral position, the lateral velocity and the lateral acceleration are comprehensively obtained. The main function of each block in the two newly developed structures is described as follows. Two angle controllers were used to eliminate the redundant components of the front-wheel steering angles. A lane scheduled gain (LSG) in each system was used to improve the lane angle deflection in UCI and UCII while the feed-forward controller simulates the behavior of a driver. The use of an empirical pre-filter reduces the lane angle error for UCI and UCII hence enhancing the performance of the system. Finally, the numerical calculation has shown that the two proposed systems are capable of tracking the desired course accurately.

Index Terms: unmanned cars, lane scheduled gains, two angle controllers and feed-forward controller.
\end{abstract}

\section{INTRODUCTION}

$\mathrm{T}$ HE development of vehicle technology has greatly increased the level of motoring convenience and in particular the popularity of front-wheeled steering (FWS) vehicles. Therefore, the important objectives of the unmanned steering control design are: (1) to provide cars with the ability to track a stated trajectory, including bends and straight roads; and (2) to minimize system error between the desired and actual signals.

Several steering control systems and strategies [1-6], which use the two degrees of freedom (2DOF) vehicle model, have been proposed. Some researchers [1][2][6] adopted the vehicle dynamics model with one input (e.g. the front-wheel steering angle), while others [3][6] considered the vehicle model with two inputs (e.g. the all-wheeled steering angle). Ackermann et al. [1] successfully described the Controlled Car I and Car II. Each of the cars was fitted with a pre-filter. While Controlled Car I uses the yaw rate gain as a steady pre-filter, Controlled Car II takes into consideration a first-order scheduled gain as the experimental pre-filter. The latter was found to have a better system performance when compared to the former. Ackermann [2] also proposed a FWS

\footnotetext{
Manuscript submitted March 8, 2006. The work is supported by National Science Council, Taiwan, R.O.C. under Grant No.94-2213-E-009-066.

B. F. Wu, is with the National Chiao Tung University, Hsinchu, Taiwan (corresponding author to provide phone: 886-03-5712121-54313; fax: 886-3-5715998; e-mail: bwu@ cssp.cn.nctu.edu.tw).

S.M. Chang, is with National Chiao Tung University, Hsinchu, Taiwan. (e-mail: smchang@cssp.cn.nctu.edu.tw).
}

system for control application. The active steering system employed was composed of a conventional steering angle $\delta_{s}$ supplied by the driver, and an additive steering angle $\delta_{C}$ provided by the controller.

Another control structure with a scheduled gain is capable of adjusting the handling angle to reduce any deflection was described by Nise [3] in his unmanned free-submersible vehicle system. For ground vehicle control application, this structure can be effectively used but the target path command cannot be taken into consideration in further research. Chen et al. [4] further proposed a system with a dynamic distance for the look-ahead scheme. The steering system with an active distance can be simplified to achieve a constant gain and thus a steering controller design which is effective regardless of the forward velocity can be obtained.

However, with respect to the automatic steering control, all the above work considered the steering angle or handling angle as an exogenous input. Based on the unmanned steering control concept, the vehicle is required to follow a programmed guideline completely when the mass and velocity vary accordingly. Hence in this study, two integrated systems which contain the feedback signals, two steering angle controllers, an empirical pre-filter, an actuator model, the lane angle scheduled (LSG) gain and the feed-forward controller have been developed. Within the two structures, the empirical pre-filter adjusts the system's performance and the two steering controllers address the unnecessary FWS angles. The lane angle deflection in each system is obtained by using the integrator model. Finally, the driver behavior is simulated by the feed-forward controller, FFC, which derives information from the lateral acceleration of the front wheels. The function of the lane angle scheduled gain can modulate the lane angle $\phi$ response so that its deflection in the two systems can be minimized.

The remainder of this paper is organized into six sections: Section II describes the Modeling, which contains the translational and rotational systems. The design maneuver which contains two additional steering angle controller designs, an empirical pre-filter and a lane angle scheduled gain designs are described in Section III. Section IV discusses the general lane maneuver. The numerical simulation is performed in Section V while the final section presents the conclusions of this study.

\section{Modeling}

This study considers a regular 2DOF linear dynamics model derived from a basic bicycle model as shown in Figure 
1: the two front wheels and the two rear wheels of the vehicle are combined into a single wheel at the center axle of each side. Therein, $V_{x}, V_{y}$ and $\gamma$ are the longitudinal velocity, the lateral velocity, and the yaw rate respectively; $\delta_{F}$ represents the FWS angle; $\beta$ is the vehicle sideslip angle; $L_{f}$ and $L_{r}$. represent the distances from the $C G$. to the front and rear axles of the vehicle, respectively; $L$ denotes the wheelbase which is the sum of the front and rear axle; $m$ is the total vehicle mass; $I$ denotes the yaw moment of inertia; $C_{f}$ and $C_{r}$ represent the total cornering stiffness of the front and rear tires, respectively.

The motion equations of the vehicle are divided into two divisions: the translational and the rotational systems. The translational system in the horizontal plane is:

$$
m a_{y}=\sum F_{y},
$$

where $\sum F_{y}$ denotes the sum of the lateral forces in the $\mathrm{y}$ direction and $a_{y}$ is the acceleration of the body. The rotational system is

$$
I \dot{\gamma}=\sum M_{f}-\sum M_{r}
$$

where $\sum M_{f}$ and $\sum M_{r}$ represent the sum of moments of the front and rear wheels about the yaw (vertical) axis, respectively. From Figure 1, the lateral and yaw motions can be expressed using the two above expressions:

$$
\begin{gathered}
m\left(\dot{V}_{y}+U \gamma\right)=2 C_{f} \alpha_{f}+2 C_{r} \alpha_{r}, \\
I \dot{\gamma}=2 L_{f} C_{f} \alpha_{f}-2 L_{r} C_{r} \alpha_{r} .
\end{gathered}
$$

Equations (3) and (4) are derived from the 2DOF linear dynamics model. The wheel-slip angle $\alpha$ of the tire is based on the following two assumptions. Firstly, if it is assumed that $V_{x} \gg V_{y}$, then the forward velocity is given by $U=\sqrt{V_{x}^{2}+V_{y}^{2}}$. Secondly, if the variation of the sideslip angle $\beta$ is sufficiently small, then $\beta=\tan ^{-1}\left(V_{y} / V_{x}\right) \approx V_{y} / V_{x}$ and its differentiation is $\dot{\beta}=\dot{V}_{y} / V_{x}$. The wheel-slip angle at the front wheels $\alpha_{f}$ and rear wheels $\alpha_{r}$ can be expected to be approximately

$$
\alpha_{f}=\delta_{F}-\beta_{f}=\delta_{F}-\tan ^{-1}\left(\frac{V_{y}+L_{f} \gamma}{V_{x}}\right) \approx \delta_{F}-\frac{V_{y}+L_{f} \gamma}{V_{x}}
$$

and

$$
\alpha_{r}=\delta_{R}-\beta_{r}=-\tan ^{-1}\left(\frac{V_{y}-L_{r} \gamma}{V_{x}}\right) \approx-\frac{V_{y}-L_{r} \gamma}{V_{x}}
$$

where $\beta_{f}$ and $\beta_{r}$ represent the chassis sideslip angle of the front tires and rear tires, respectively (Note that: $\delta_{R} \equiv 0$, for FWS vehicle). The tire model is summarized in Table I. In the translational system, the acceleration of the body is represented by $a_{\mathrm{CG}}=\dot{V}_{y}+U \gamma=U(\dot{\beta}+\dot{\varphi})=U \dot{\phi}$. Using this equation, it was deduced that the product of the forward velocity, $U$, and the lane rate, $\dot{\phi}$, are equivalents to the lateral acceleration at the body. The lane angle, $\phi$, is obtained from the first integral of $a_{\text {CG. }}$ i.e. $\phi=\beta+\varphi$. The differentiation of $\phi$ gives

$$
\begin{aligned}
\dot{\phi} & =\dot{\beta}+\gamma \\
& =\left(a_{11} \beta+a_{12} \gamma+b_{11} \delta_{F}\right)+\gamma \\
& =a_{11} \beta+\left(1+a_{12}\right) \gamma+b_{11} \delta_{F} .
\end{aligned}
$$

By combining equations (3)-(7) and then transforming them into the state-space model gives:

$$
\left[\begin{array}{c}
\dot{\beta} \\
\dot{\gamma} \\
\dot{\phi}
\end{array}\right]=\left[\begin{array}{ccc}
a_{11} & a_{12} & 0 \\
a_{21} & a_{22} & 0 \\
a_{11} & 1+a_{12} & 0
\end{array}\right]\left[\begin{array}{l}
\beta \\
\gamma \\
\phi
\end{array}\right]+\left[\begin{array}{l}
b_{11} \\
b_{21} \\
b_{11}
\end{array}\right] \delta_{F},
$$

where

$$
\begin{gathered}
a_{11}=-\frac{2\left(C_{f}+C_{r}\right)}{m U}, a_{12}=-1-\frac{2\left(L_{f} C_{f}-L_{r} C_{r}\right)}{m U^{2}}, a_{21}=-\frac{2\left(L_{f} C_{f}-L_{r} C_{r}\right)}{I}, \\
a_{22}=-\frac{2\left(L_{f}{ }^{2} C_{f}+L_{r}{ }^{2} C_{r}\right)}{I U}, b_{11}=\frac{2 C_{f}}{m U} \text { and } b_{21}=\frac{2 L_{f} C_{f}}{I} .
\end{gathered}
$$

Since the relationship of the steering angle, $\delta_{C}$, and the conventional steering angle, $\delta_{s}$ have been proposed by Ackermann [2], in this study the relationship $\delta_{F}=\delta_{S C_{1}}+\delta_{S C_{2}}+\delta_{C}+\delta_{S}$ is first considered and then substituted into (8) as

$$
\left[\begin{array}{l}
\dot{\beta} \\
\dot{\gamma} \\
\dot{\phi}
\end{array}\right]=\left[\begin{array}{ccc}
a_{11} & a_{12} & 0 \\
a_{21} & a_{22} & 0 \\
a_{11} & 1+a_{12} & 0
\end{array}\right]\left[\begin{array}{l}
\beta \\
\gamma \\
\phi
\end{array}\right]+\left[\begin{array}{llll}
b_{11} & b_{11} & b_{11} & b_{11} \\
b_{21} & b_{21} & b_{21} & b_{21} \\
b_{11} & b_{11} & b_{11} & b_{11}
\end{array}\right]\left[\begin{array}{l}
\delta_{S C_{1}} \\
\delta_{S C_{2}} \\
\delta_{C} \\
\delta_{S}
\end{array}\right],
$$

where $\delta_{S}$ is generated by the driver command, $\delta_{C}$ is produced by the actuator model and both $\delta_{S C_{1}}$ and $\delta_{S C_{2}}$ are the feedback controller signals supplied respectively by the two angle controllers $\mathrm{T}_{\text {contr. I }}$ and $\mathrm{T}_{\text {contr.II }}$. Incorporating the two variable relationships, $\dot{\varphi}=\gamma$ and $\dot{y}_{L}=U \phi$, into (9) gives

$$
\left[\begin{array}{l}
\dot{\beta} \\
\dot{\gamma} \\
\dot{\phi} \\
\dot{\varphi} \\
\dot{y}_{L}
\end{array}\right]=\left[\begin{array}{lllll}
a_{11} & a_{12} & 0 & 0 & 0 \\
a_{21} & a_{22} & 0 & 0 & 0 \\
a_{11} & 1+a_{12} & 0 & 0 & 0 \\
0 & 1 & 0 & 0 & 0 \\
0 & 0 & U & 0 & 0
\end{array}\right]\left[\begin{array}{l}
\beta \\
\gamma \\
\phi \\
\varphi \\
y_{L}
\end{array}\right]+\left[\begin{array}{llll}
b_{11} & b_{11} & b_{11} & b_{11} \\
b_{21} & b_{21} & b_{21} & b_{21} \\
b_{11} & b_{11} & b_{11} & b_{11} \\
0 & 0 & 0 & 0 \\
0 & 0 & 0 & 0
\end{array}\right]\left[\begin{array}{c}
\delta_{S C_{1}} \\
\delta_{S C_{2}} \\
\delta_{C} \\
\delta_{S}
\end{array}\right] .
$$

where $\varphi$ denotes the handling angle and $y_{L}$ indicates the lateral position at $\mathrm{CG}$.

\section{DESign Maneuver}

The section describes the methods in which the two additional angle controllers, the lane scheduled gain, the empirical pre-filter and the feed-forward controller for each steering structure were designed. Two additional angle controllers are necessary if the residue component of $\delta_{F}$ is to be eliminated. One of the angle controllers $\mathrm{T}_{\text {contr. } \mathrm{I}}$ can be designed using the 2DOF model; the other controller $\mathrm{T}_{\text {contr.II }}$ can be calculated using the front-axle concept. The lane scheduled gain, as the name suggests, is fed with the lane angle signal for reducing the yaw rate and sideslip angle errors of the system for two frameworks. The difference between the two proposed systems is that the latter system includes the handling gain while the former does not. Finally, the feed-forward controller, derived from the formulated lateral acceleration with an active distance at the front axle, shows that the relationship between $r_{\text {course }}$ and $\delta_{S}$, and demonstrates conclusively the effective simulation of human behavior. 


\section{A. Additional angle controller descriptions}

From (10), the vehicle model with the steering angle signal $\delta_{S C_{1}}$ is

$$
\left[\begin{array}{l}
\dot{\beta} \\
\dot{\gamma} \\
\dot{\phi}
\end{array}\right]=\left[\begin{array}{ccc}
a_{11} & a_{12} & 0 \\
a_{21} & a_{22} & 0 \\
a_{11} & 1+a_{12} & 0
\end{array}\right]\left[\begin{array}{l}
\beta \\
\gamma \\
\phi
\end{array}\right]+\left[\begin{array}{l}
b_{11} \\
b_{21} \\
b_{11}
\end{array}\right] \delta_{S C_{1}} .
$$

The angle controller $1, \mathrm{~T}_{\text {contr. }}$, can be obtained by setting the sideslip angle, $\beta$, to zero and the following expression is obtained as

$$
-a_{12} \gamma=b_{11} \delta_{S C_{1}} \Leftrightarrow \delta_{S C_{1}}=-b_{11}^{-1} a_{12} \gamma=\mathrm{T}_{\text {contr.I }} \gamma,
$$

where

$$
\mathrm{T}_{\text {contr. } \mathrm{I}}=\frac{m U^{2}+2\left(C_{f} L_{f}-C_{r} L_{r}\right)}{2 C_{f} U} .
$$

From (12), $\mathrm{T}_{\text {cont.l. }}$ contains the unessential front-wheel component. In order to eliminate $C_{f} L_{f}$, the second steering controller needs to be taken into account as

$$
\delta_{S C_{2}}=\mathrm{T}_{\text {contr.II }} \gamma \text {, }
$$

where

$$
\mathrm{T}_{\text {contr.II }}=L_{f} / U
$$

Equations (12) and (13) are substituted into (10) to yield

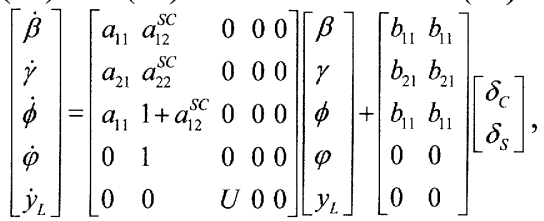

where $a_{12}^{s c}=a_{12}+b_{11}\left(\mathrm{~T}_{\text {contr.I }}-\mathrm{T}_{\text {contr.II }}\right)$ and $a_{22}^{s c}=a_{22}+b_{21}\left(\mathrm{~T}_{\text {contr.I }}-\mathrm{T}_{\text {contr.II }}\right)$.

\section{B. Empirical pre-filter and Lane angle scheduled gain designs}

The empirical pre-filter and the lane angle scheduled gain in each system are designed. For UCI,

$$
\begin{aligned}
\dot{\delta}_{c} & =-\mathrm{T}_{\mathrm{LSG}_{1}} \phi+\gamma_{r s} \\
& =-\mathrm{T}_{\mathrm{ISG}_{1}} \phi+\mathrm{T}_{\mathrm{E}_{-\mathrm{pref}}} \delta_{S},
\end{aligned}
$$

where $\mathrm{T}_{\mathrm{LSG}_{\mathrm{I}}}$ and $\mathrm{T}_{\mathrm{E}-\mathrm{pref}}$ denote the transfer function of the lane angle scheduled gain and that of the empirical pre-filter, respectively. By substituting (15) into (14), the state-space model is extended to

$$
\left[\begin{array}{l}
\dot{\beta} \\
\dot{\gamma} \\
\dot{\phi} \\
\dot{\varphi} \\
\dot{y}_{L} \\
\dot{\delta}_{C}
\end{array}\right]=\left[\begin{array}{llllll}
a_{11} & a_{12}^{S C} & 0 & 0 & 0 & b_{11} \\
a_{21} & a_{22}^{S C} & 0 & 0 & 0 & b_{21} \\
a_{11} & 1+a_{12}^{S C} & 0 & 0 & 0 & b_{11} \\
0 & 1 & 0 & 0 & 0 & 0 \\
0 & 0 & U & 0 & 0 & 0 \\
0 & 0 & -\mathrm{T}_{\mathrm{IS}_{\mathrm{I}}} & 0 & 0 & 0
\end{array}\right]\left[\begin{array}{l}
\beta \\
\gamma \\
\phi \\
\varphi \\
y_{L} \\
\delta_{C}
\end{array}\right]+\left[\begin{array}{l}
b_{11} \\
b_{21} \\
b_{11} \\
0 \\
0 \\
\mathrm{~T}_{\mathrm{E}_{\mathrm{pref}}}
\end{array}\right] \delta_{S} .
$$

For UCII, in order to enhance the maneuverability of the vehicle, the handling angle signal $\varphi$ and the handling gain $\mathrm{T}_{\mathrm{HG}_{\|}}$are added as follows:

$$
\begin{aligned}
& \dot{\delta}_{c}=-\mathrm{T}_{\mathrm{LSG}_{\mathrm{II}}} \phi+\gamma_{r s} \\
& =-\mathrm{T}_{\mathrm{LSG}_{\mathrm{II}}} \phi+\mathrm{T}_{\mathrm{HG}_{\mathrm{II}}}\left(\varphi_{d}-\varphi\right) \\
& =-\mathrm{T}_{\mathrm{LSG}_{\text {III }}} \phi+\mathrm{T}_{\mathrm{HG}_{\mathrm{II}}}\left(\mathrm{T}_{\mathrm{E}_{\mathrm{pref}} \mathrm{II}} \delta_{S}-\varphi\right) \text {, }
\end{aligned}
$$

where $\varphi_{d}$ represents the desired handling angle and $\mathrm{T}_{\mathrm{E} \text { pref }_{\mathrm{II}}}$ and $T_{L_{S G}}$ denote the transfer functions of the empirical pre-filter and the lane angle scheduled gain. Similarly, by merging (17) into (14), the following is obtained:

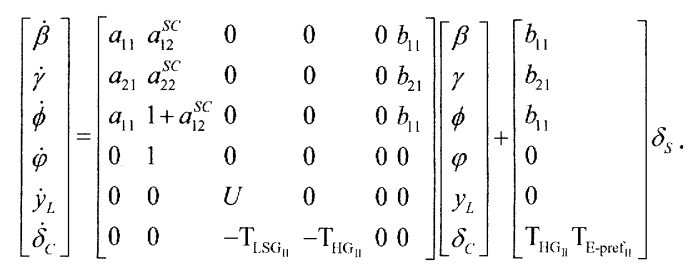

Furthermore, the empirical pre-filter $\mathrm{T}_{\mathrm{E} \text {.pref }}$ and the lane angle scheduled gain $T_{L S G}$ for each system are chosen as

$$
\mathrm{T}_{\text {E.pref }}=\left\{\begin{array}{c}
13, \text { for UCI } \\
1400, \text { for UCII }
\end{array}\right.
$$

and

$$
\mathrm{T}_{\mathrm{LSG}}=\left\{\begin{array}{l}
1.7, \text { for } \mathrm{UCI} \\
1.8, \text { for UCII }
\end{array}\right.
$$

and the handling angle gain used in $\mathrm{UCII}$ is $\mathrm{T}_{\mathrm{HG}_{11}}=0.01$.

\section{General Lane Manner}

The feed-forward controller FFC, depicted in Figures 3 and 4 , can be deduced from the vehicle motion model using a PI filter. By extracting the vehicle model from (18) with the sideslip angle, the yaw rate, the lane angle, and the lateral position, the following equation is obtained:

$$
\left[\begin{array}{c}
\dot{\beta} \\
\dot{\gamma} \\
\dot{\phi} \\
\dot{y}_{L}
\end{array}\right]=\left[\begin{array}{cccc}
a_{11} & a_{12}^{S C} & 0 & 0 \\
a_{21} & a_{22}^{S C} & 0 & 0 \\
a_{11} & 1+a_{12}^{S C} & 0 & 0 \\
0 & 0 & U & 0
\end{array}\right]\left[\begin{array}{c}
\beta \\
\gamma \\
\phi \\
y_{L}
\end{array}\right]+\left[\begin{array}{c}
b_{11} \\
b_{21} \\
b_{11} \\
0
\end{array}\right],
$$

and then the relationship between $\delta_{s}$ and $\gamma$ is obtained from (21) as

$$
\frac{\gamma(s)}{\delta_{S}(s)}=\frac{N_{\gamma}(s)}{D(s)}
$$

where the numerator is

$$
N_{\gamma}(s)=2 C_{f} U\left[m L_{f} U s+2 C_{r} L\right],
$$

and the denominators is

$$
\begin{aligned}
D(s)=m I U^{2} \mathrm{~s}^{2}+ & {\left[2 m\left(L_{f}^{2} C_{f}+C_{r} L_{r} L\right)+2 I\left(C_{f}+C_{r}\right)-m^{2} U^{2} L_{f}\right] U \mathrm{~s} } \\
+ & 2\left[2 L_{r} C_{r}^{2} L+2 C_{f} L^{2} C_{r}-m U^{2} L_{f}\left(C_{f}+C_{r}\right)\right] .
\end{aligned}
$$

In addition, the relationship between $\delta_{s}$ and $\phi$ is derived from (21) giving

$$
\frac{\phi(s)}{\delta_{S}(s)}=\frac{N_{\phi}(s)}{D_{\phi}(s)},
$$

where the numerator expresses

$$
N_{\phi}(s)=2 C_{f}\left[U^{2} I s^{2}+2 C_{r} L_{r} L s+2 C_{r} U L\right],
$$

and the denominator represents $D_{\phi}(s)=s \cdot D(s)$.

The formulated lateral acceleration of the body $a_{\mathrm{CG}}$ and the front axle $a_{f}$ can be described in equations (24) and (25) respectively as follows:

$$
a_{\mathrm{CG} .}=U \dot{\phi},
$$

and

$$
a_{f}=a_{\mathrm{CG} .}+L_{f} \dot{\gamma}
$$

From (25), an additional term $L_{f} \dot{\gamma}$, the acceleration component in the lateral direction at the front axle, is shown to be necessary if the front-axle lateral acceleration $a_{f}$ is to be calculated. Based on the front-axle lateral acceleration 
concept, an active distance [5] $L_{d f}$, substituted for an inactive distance $L_{f}$, is considered in the time domain as

$$
L_{d f}(t)=c_{1} \delta(t)+c_{2},
$$

where $\delta(t)$ represents the unit impulse function; $c_{1}$ and $c_{2}$ need to be determined. The lateral acceleration with (26) at the front wheels can be rewritten as

$$
a_{f}=a_{\mathrm{CG}}+L_{d f} \dot{\gamma} .
$$

Using Laplace transformation in (24), (26) and (27), the following equations are obtained as

$$
\begin{gathered}
a_{\mathrm{CG} .}(s)=U s \cdot \phi(s), \\
L_{d f}(t)=c_{1}+\frac{c_{2}}{s}
\end{gathered}
$$

and

$$
a_{f}(s)=a_{\mathrm{CG} .}(s)+s \cdot L_{d f}(s) \gamma(s) .
$$

Then, by substituting (28) (29) into (30), the following is expressed:

$$
a_{f}(s)=U s \cdot \phi(s)+s \cdot\left(c_{1}+\frac{c_{2}}{s}\right) \gamma(s) .
$$

Substituting (22) and (23) into (31), the relationship, $a_{f}(s) / \delta_{S}(s)$, is obtained:

$$
\frac{a_{f}(s)}{\delta_{S}(s)}=\frac{U N_{\phi}(s)+\left(c_{1} s+c_{2}\right) N_{\gamma}(s)}{D(s)}=\frac{1}{s^{2}} \cdot \frac{N_{a f}(s)}{D(s)},
$$

where the numerator represents

$$
N_{u f}(s)=2 U C_{f}\left[\left(U^{2} I+m L_{f} U c_{1}\right) s^{2}+\left(2 C_{r} L_{r} L+m L_{f} U c_{2}+2 C_{r} L c_{1}\right) s+2 C_{r} U L+2 C_{r} L c_{2}\right]
$$

and the same denominator $D(s)$ expressed in (22) is repeated. The redefined front-axle lateral acceleration in (32) can be simplified as a constant gain $k_{a f}$ if the two parameters $c_{1}$ and $c_{2}$ placed at $N_{a f}(s)$ in (32) are determined. In order to determine $L_{d f}(s)$, the numerator $N_{a f}(s)$ is assumed to be equal to the product of a gain $k_{a f}$ and the denominator $D(s)$, that is, $N_{a f}(s)=k_{a f} \cdot D(s)$. The two following equations, based on this expression, can be used to determine parameters $c_{1}$ and $c_{2}$ as

$$
2 U C_{f}\left(U^{2} I+m L_{f} U c_{1}\right)=k_{d f} m I U^{2},
$$

and

$$
2 U C_{f}\left(2 C_{r} U L+2 C_{r} L c_{2}\right)=2 k_{d f}\left[2 L_{r} C_{r}^{2} L+2 C_{f} L^{2} C_{r}-m U^{2} L_{f}\left(C_{f}+C_{r}\right)\right] .
$$

From (33), the parameter $c_{1}$ is obtained

$$
c_{1}=k_{d f} \frac{I}{2 C_{f} L_{f}}-\frac{U I}{m L_{f}} .
$$

and parameter $c_{2}$ can be obtained from (34) as

$$
c_{2}=k_{d f} \frac{2 L_{r} C_{r}^{2} L+2 C_{f} L^{2} C_{r}-m U^{2} L_{f}\left(C_{f}+C_{r}\right)}{2 C_{r} L U C_{f}}-U .
$$

By substituting $c_{1}$ and $c_{2}$ into $N_{a f}(s)$ in (32) and rewriting it gives

$$
\begin{aligned}
& N_{a f}(s)=2 U C_{f}\left(U^{2} I+m L_{f} U\left[\frac{l}{2 l_{f} C_{f}} k_{d f}-\frac{1}{m L_{f}}\right]\right) s^{2}
\end{aligned}
$$

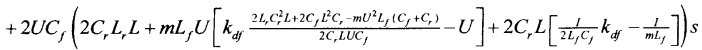

$$
\begin{aligned}
& +2 U C_{f}\left(2 C_{r} U L+2 C_{r} L\left[k_{d f} \frac{2 L C_{l}^{2} L+2 C_{f}, L_{C}^{2},-m U^{2} L_{f},\left(C_{f}+C_{r}\right)}{2 C_{,}, U C_{f}}-U\right]\right)
\end{aligned}
$$

The product of the gain $k_{d f}$ and the coefficient of term $S$ in $D(s)$ is equivalent to the coefficient of term $S$ in $N_{d f}(s)$ as follows:

$$
\begin{aligned}
& 2 U C_{f}\left(2 C_{r} L_{r} L+m L_{f} U\left[k_{d f} \frac{2 l, r_{l}^{2} L+2 C_{f} C_{C} C_{r}-m C^{2} L_{f}\left(C_{f}+C_{r}\right)}{2 C_{,} U U C_{f}}-U\right]+2 C_{r} L\left[\frac{1}{2 L l_{f} C_{f}} k_{d f}-\frac{I}{m L_{f}}\right]\right) \\
& =k_{d f}\left[2 m\left(L_{f}^{2} C_{f}+C_{r} L_{r} L\right)+2 I\left(C_{f}+C_{r}\right)-m^{2} U^{2} L_{f}\right] U
\end{aligned}
$$

Therefore, $k_{d f}$ can be determined as

$$
k_{d f}=\frac{2 C_{f} C_{r} L}{m\left(C_{r} L_{r}-L_{f} C_{f}\right)},
$$

and when substituted it into (16) and (18) yields the two following equations

$$
\left[\begin{array}{l}
\dot{\beta} \\
\dot{\gamma} \\
\dot{\phi} \\
\dot{\varphi} \\
\dot{y}_{L} \\
\dot{\delta}_{C}
\end{array}\right]=\left[\begin{array}{llllll}
a_{11} & a_{12}^{S C} & 0 & 0 & 0 & b_{11} \\
a_{21} & a_{22}^{S C} & 0 & 0 & 0 & b_{21} \\
a_{11} & 1+a_{12}^{S C} & 0 & 0 & 0 & b_{11} \\
0 & 1 & 0 & 0 & 0 & 0 \\
0 & 0 & U & 0 & 0 & 0 \\
0 & 0 & -\mathrm{T}_{\mathrm{LSG}_{1}} & 0 & 0 & 0
\end{array}\right]\left[\begin{array}{l}
\beta \\
\gamma \\
\phi \\
\varphi \\
y_{L} \\
\delta_{C}
\end{array}\right]+\left[\begin{array}{l}
b_{11} \\
b_{21} \\
b_{11} \\
0 \\
0 \\
\mathrm{~T}_{\mathrm{E}-\mathrm{pref}}
\end{array}\right] k_{d f} r_{\text {course }},
$$

and

$$
\left[\begin{array}{l}
\dot{\beta} \\
\dot{\gamma} \\
\dot{\phi} \\
\dot{\varphi} \\
\dot{y}_{L} \\
\dot{\delta}_{C}
\end{array}\right]=\left[\begin{array}{llllll}
a_{11} & a_{12}^{S C} & 0 & 0 & 0 & b_{11} \\
a_{21} & a_{22} & 0 & 0 & 0 & b_{21} \\
a_{11} & 1+a_{12} & 0 & 0 & 0 & b_{11} \\
0 & 1 & 0 & 0 & 0 & 0 \\
0 & 0 & U & 0 & 0 & 0 \\
0 & 0 & -\mathrm{T}_{\mathrm{LSG}_{11}}-\mathrm{T}_{\mathrm{HG}_{\mathrm{H}}} & 0
\end{array}\right]\left[\begin{array}{l}
\beta \\
\gamma \\
\phi \\
\varphi \\
y_{L} \\
\delta_{C}
\end{array}\right]+\left[\begin{array}{l}
b_{11} \\
b_{21} \\
b_{11} \\
0 \\
0 \\
\mathrm{~T}_{\mathrm{HG}_{\mathrm{II}}} \mathrm{T}_{\mathrm{E}_{- \text {pref }_{11}}}
\end{array}\right] k_{d f} r_{\text {course }},
$$

where $r_{\text {course }}$ denotes the input command. Equations (37) and (38) can further be transferred into the two schematic diagrams as illustrated in Figure 3 and 4.

\section{Simulation Results}

In this section, the system performance is illustrated as follows. Figures 6-11 depict the upgraded plot with the solid lines and the degraded plot with the dotted lines for the system's performance. Figures 12(a) and 12(b) illustrate the errors in the lane angle, $e_{\text {lane-ang }}$, which can be obtained by using the first integral of the error in the lane rate.

Furthermore, Figure 6, which illustrates the sideslip angle response of the body (the upper plot), front-wheel (the left-down plot) and rear-wheel (the right-down plot) positions, describes the tire variation behaviors while the vehicle travels on the designated lane. Figure 7 shows the slip angle at frontand rear- wheel places, located at the upper and down plots respectively, while the presence and absence of LSG are considered in the two steering structures. The slip angle responses of the rear wheels, placed at the down plot in Figure 7, can be obtained by using the formula expression $\alpha_{r}=-\beta_{r}$. Similarly, the slip angle responses of the front wheels, located at the upper plot in Figure 7, can be represented by following the expression, $\alpha_{f}=\delta_{F}-\beta_{f}$.

Figure 8 shows the responses of the handling angle, $\varphi$, the yaw rate, $\gamma$ and the steering angle, $\delta_{F}$, respectively, between the presence and absence of LSG. The handling angle response represented with the solid lines obviously outperforms that represented with the dotted lines. The yaw rate response, located at the left-down plot in Figure 8, with LSG ranges from - 33 to 33 degrees/seconds for two cases. By contrast, the yaw rate plot without LSG is limited to a range within 50 degrees/seconds for two cases. The steering angle response with the solid lines ranges from -9 to 9 degrees and with the dotted lines locates between -14 and 14 degrees. In reality, when a vehicle moves along a designated road, the 
yaw rate response is directly related with the steering wheel within the forward velocity, mass and tire-road contact variations.

Figure 9 illustrates the lateral acceleration response at the body, front-wheel and rear-wheel positions. The magnitude in Figure 9 is located between $-0.7 \mathrm{~g}$ and $0.7 \mathrm{~g}$ while LSG is employed in each system. The downgraded performance in Figure 9, denoted with the dotted lines, illustrates the absence of LSG. For application, the lateral acceleration has a reasonable range from $-\mathrm{g}$ to $\mathrm{g}$ while the vehicle moves along the presented road. Based on this permissible range, the upgraded response is preferable to the downgraded response. Figure 10 depicts the lateral displacement responses with and without LSG. The responses, placed at the body, front-wheel and rear-wheel positions, with LSG have the same waveform as the given command. The degraded lateral position response, denoted by the dotted lines, has an abnormal range based on the presented command. The responses of the actual lateral acceleration in Figure 9 and the actual lateral position in Figure 10 can be illustrated with the lane angle so the actual lateral velocity, shown in Figure 11, can be obtained. Among Figures 9-11, the physical relationship can be represented as follows: the first differentiation of the lateral position response is equivalent to the lateral velocity response while the second differentiation of the lateral position response is equivalent to the lateral acceleration response. Figures 12(a) and 12(b) illustrate the error of the lane angle $e_{\text {lane-ang }}$, which represented by the solid lines. By contrast, the degraded response in Figures 12(a) and 12(b) represent by the dotted lines. Obviously, the two steering systems directed with the actual command have an approximate error range in the lane angle. In short, it was observed that the whole system performance with LSG was better than that without LSG.

\section{CONClusions}

The two developed structures allow the vehicle to remain on its lane. Both the structures were found to be effective. UCI uses one gain, the lane scheduled gain, two feedback signals, the lane and yaw rates. On the other hand, UCII adopts two gains, the lane scheduled and handling gains, three feedback signals, the handling angle, the lane and yaw rates. The two angle controllers are capable of compensating for the redundant FWS angle component. The feed-forward controller can successfully simulate the driver command. This study of the vehicle steering system demonstrates the three main points: (1) the response of the steering angle shares the same waveform as the yaw rate; (2) the lateral acceleration for the application can be located within a reasonable range, while the vehicle travels on the actual lane command; (3) the lane angle error can be minimized by controlling the empirical pre-filter and the lane scheduled gain. The focus of the future study will be the advanced steering control structure, which contains of the roll dynamic model, with the road curvature.

\section{ACKNOWLEDGMENT}

The work is supported by National Science Council, Taiwan, R.O.C. under NSC.94-2213-E-009-066.

\section{REFERENCES}

[1] J. Ackermann, and T. Bünte, "Handling Improvement for robustly decoupled car steering dynamics", in Proc. of the $4^{\text {th }}$ IEEE Mediterranean Symposium on New directions in Control and Automation, 1996, pp. 83-88 (Chania, Crete, Greece)..

[2] J. Ackermann, "Robust control prevents car skidding", IEEE Control Systems Magazine, 1997, pp. 23-31.

[3] N. S. Nise, Control Systems Engineering, $2^{\text {nd }}$ edition, Addison-Wesley Publishing 1995.

[4] C. Chen, and H. S. Tan, "Steering Control of High Speed Vehicles: Dynamic Look Ahead and Yaw Rate Feedback", in Proc. of the $37^{\text {th }}$ IEEE Conference on Decision \& Control (Tampa, Florida USA), pp. 1025-10301998.

[5] S. Tsugawa, H. Mori, and S. Kato, "A Lateral Control Algorithm for Vision-Based Vehicles with a Moving Target in the Field of View", IEEE international Conference on Intelligent Vehicles, pp. 41-45 1998.

[6] F. Yoshimi, Y. Naohiro, S. Shoichi, T. Hideo, and M. Yoshinobu, "A Review of Four-Wheel Steering Studies from the Viewpoint of Vehicle Dynamics and Control", Vehicle System Dynamics, vol. 18, pp. 151-186, 1989.

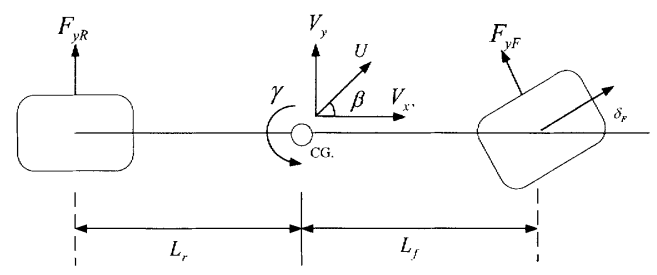

Figure 1: The equivalent dynamics model for vehicle.

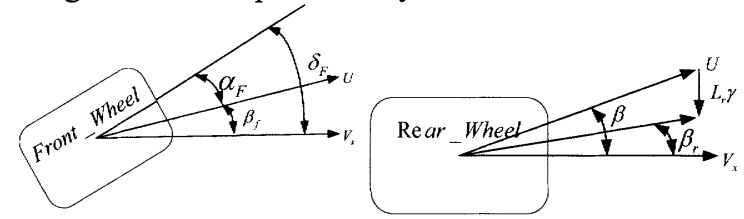

Figure 2: Tire angles.

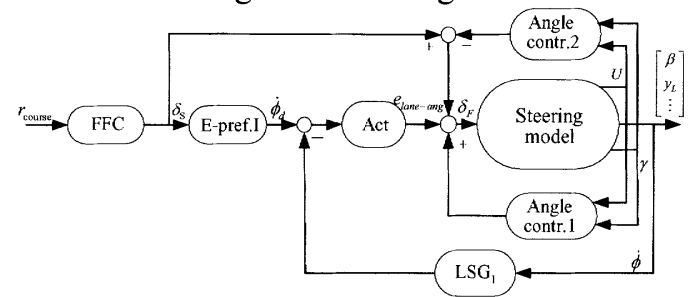

Figure 3: Unmanned Car I

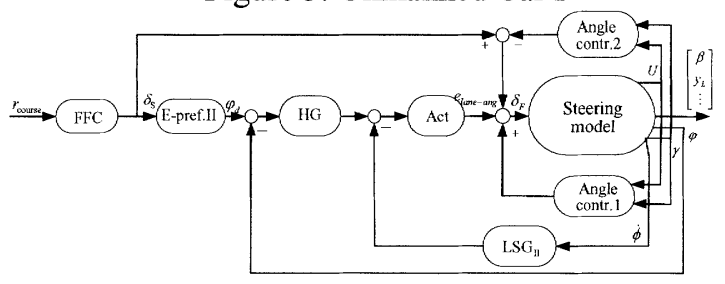

Figure 4: Unmanned Car II 


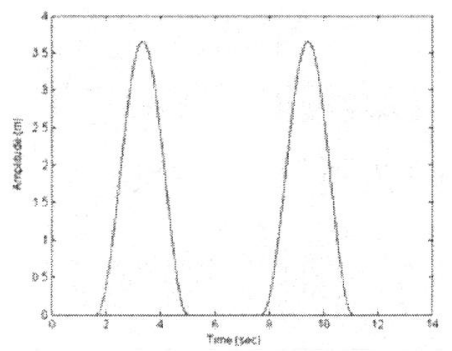

Figure 5: Input signal.
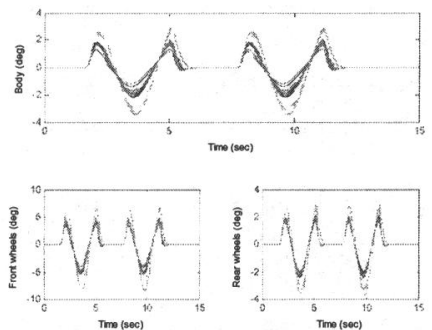

Figure 6: The upgraded and degraded plots for the sideslip angle at the body, front-wheel and rear-wheel positions.
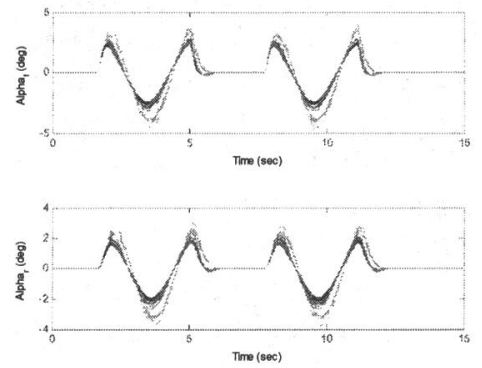

Figure 7: The upgraded and degraded plots of the slip angle response at the front- and rear-wheel positions.
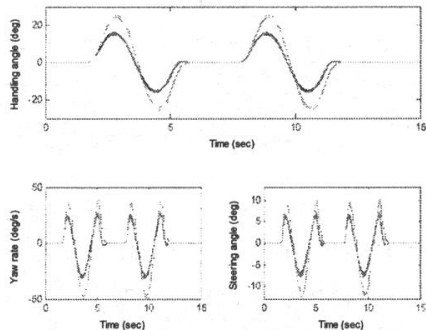

Figure 8: The upgraded and degraded plots of handling angle, yaw rate and steering angle.
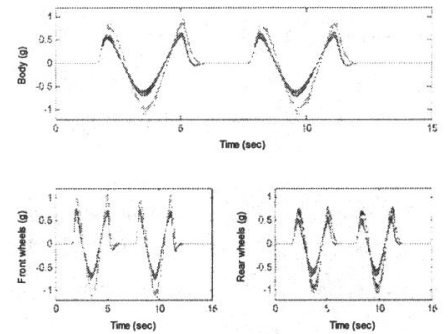

Figure 9: The upgraded and degraded plots for lateral acceleration at the body, front-wheel and rear-wheel positions.
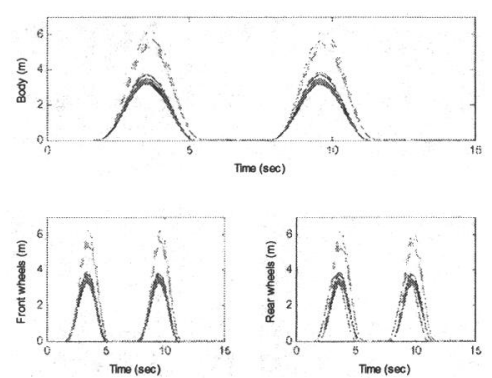

Figure 10: The upgraded and degraded plots for lateral position at the body, front- and rear- wheel positions.
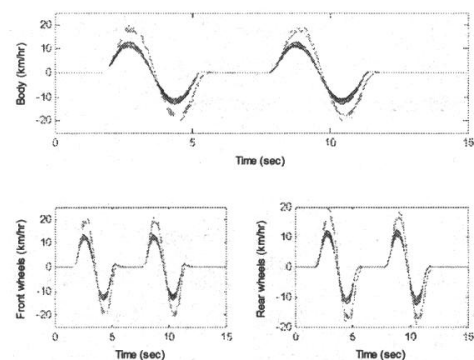

Figure 11: The upgraded and degraded plots for lateral velocity at the body, front- and rear- wheel positions.

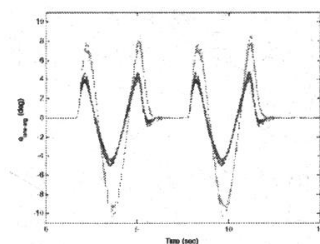

(a)

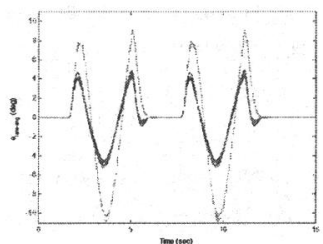

(b)
Figure 12: The upgraded and degraded plots for lane angle

TABLE I

THE TIRE ANGLE DESCRIPTIONS

\begin{tabular}{lcc}
\hline \hline \multicolumn{1}{c}{ Symbol } & Rear axles & Front axles \\
\hline Steer & 0 & $\delta_{F}$ \\
Sideslip angle $(\beta)$ & $\left(V_{y}-L_{r} \gamma\right) / V_{x}$ & $\left(V_{y}+L_{j} \gamma\right) / V_{x}$ \\
Wheel-slip $(\alpha)$ & $-\left(V_{y}-L_{r} \gamma\right) / V_{x}$ & $\delta_{F}-\left(V_{y}+L_{f} \gamma\right) / V_{x}$
\end{tabular}

$$
\text { error - (a) UCI; (b) UCII. }
$$

TABLE II

SAVRIN CAR PARAMETERS

\begin{tabular}{ccc}
\hline \multicolumn{3}{c}{ SAVRIN CAR PARAMETERS } \\
\hline \hline Symbol & Quantity (unit) & Values \\
\hline $\mathrm{m}$ & Mass $(\mathrm{kg})$ & {$[1640,2200]$} \\
$C_{f}$ & front-wheel stiffness (N/rad) & $65695.5^{*} 2$ \\
$C_{r}$ & rear-wheel stiffness (N/rad) & $57834.5^{*} 2$ \\
$L_{f}$ & front-axle distance $(\mathrm{m})$ & 1.193 \\
$L_{r}$ & Rear-axle distance $(\mathrm{m})$ & 1.583 \\
$I$ & inertia moment $\left(\mathrm{kg}-\mathrm{m}^{2}\right)$ & 2300 \\
$U$ & forward velocity $(\mathrm{km} / \mathrm{hr})$ & {$[40,55]$} \\
$\mu$ & road-tire contact & {$[0.5,1.0]$}
\end{tabular}

